\title{
A Case of Fibro-myoma of the Uterus undergoing Sarcomatous Change.*
}

BY

W. S. A. GrIFrith, M.D., Assistant Obstetric Physician to St. Bartholomew's Hospital, London,

AND

Herbert Willtauson, M.B., Demonstrator of Practical Obstetrics, St. Bartholomew's Hospital Medical School.

G.F., AGED 56, was admitted into St. Bartholomew's Hospital under the care of Dr. Herringham, on June 2rth, 1905, suffering from abdominal pain and diarrhoea.

The patient had been a widow for nineteen years. She had never been pregnant. Very little is known of her past history except that six years previously she had attended the hospital as an out-patient, on account of an abdominal tumour and of excessive losses at her menstrual periods. At that time she was seen by Dr. Champneys, who diagnosed uterine fibroids, but advised that, as she was not a fit subject, no operation should be performed.

At the beginning of May, 1905, the patient commenced to suffer from abdominal pain, cough and night-sweats; on June 25th from persistent headache, and from diarrhæa, passing 3 or 4 loose motions a day. At the time she presented herself at the hospital her temperature was $101^{\circ} \mathrm{F}$. The symptoms were suggestive of enteric fever, and she was sent into the ward with this provisional diagnosis.

On admission, her condition was thus described:-

"A stout, heavily-built woman, with no marked anæmia. Temperature $101^{\circ} \mathbf{F}$. Pulse 100 , of fair volume and tension. Respirations 28 per minute. Tongue coated in the centre, red at the edges. Nothing abnormal is discovered in heart or lungs.

"Per hypogastrium, a large firm tumour rises out of the pelvis, and reaches to two inches above the umbilicus; it lies more on the right than on the left side of the abdomen. The spleen cannot be felt.

* Read at a Meeting of the Obstetrical Society of London, held on January 3rd, 1906. For the discussion see under "Reports of Societies," in this number of the Journal. 


\section{Griffith and Williamson: Sarcomatous Fibro-myoma}

Blood-count-no diminution of red-blood corpuscles, leucocytes 11,000. The blood was tested for Widal's reaction, a dilution of 1 in 40 was used; two or three small clumps were observed but the reaction was not sufficient to justify a positive diagnosis."

On July 4th the test was re-applied, using a dilution of 1 in 20 ; there was agglutination. This was repeated on July 6 th, again with a negative result.

After admission the diarrhoa ceased, the temperature ran an irregular course, varying between $99^{\circ}$ and $101^{\circ}$; the woman was obviously ill and there was difficulty of respiration in the recumbent posture, although there were no physical signs to indicate any pathological condition in the lungs.

On July 17th, she was seen by Dr. Champneys, who made the following note:-

"Per hypogastrium. A hard tumour reaches $2 \frac{1}{2}$ inches above the navel; the abdomen is not tender.

"Per vaginam. The vagina admits one finger only, the cervix is small and high up.

"Bimanually. The abdominal mass moves with the cervix.

"Per rectum. Nothing further is discovered.

"The patient has a large fibroid which is freely moveable. The pelvic tissues feel normal; there is no reason to connect the temperature with any pelvic condition."

Between July 11th and August 8th the patient's general condition became steadily worse. The temperature remained persistently above the normal, and on several occasions rigors occurred, with a further rise of temperature to $103^{\circ}$. There were sometimes attacks of abdominal pain, and the patient complained also of pain in the chest. On July 11th peptone was detected in the urine. A note was made: "The presence of peptonuria suggests that some necrosis is going on, possibly in the fibroid." On August 2nd cultures were taken from the blood with a negative result. From August 15th to 17th hæmorrhage occurred from the vagina; a considerable quantity of blood was lost, and clots were passed. A day or two after the cessation of this hæmorrhage the patient was seen by Dr. Griffith. In view of the facts that she was getting gradually worse, and that the uterine tumour was the only pathological lesion that could be discovered, he urged that an exploratory operation should be undertaken. The patient however refused her consent.

By August 22nd the general condition was much worse, the pulse was now 120 and the respirations were 40 per minute; injections of 
10 c.c. of anti-streptococcus serum were administered twice daily. On this date the following note was made:-

"Over the right lung in front there is loss of resonance and the breath sounds are weak. Behind, there is impairment of percussion note at the right apex, the breath sounds are weak and the vocal resonance increased."

It was now suspected that the uterine tumour was of a malignant nature, and that secondary deposits were present in the lung.

From August 24 th to 29 th there was hæmorrhage from the vagina, large clots were passed each day.

The pationt then requested that the operation might be performed, and on September lst she was transferred to Martha Ward, under the care of Dr. Griffith.

Her general condition was, however, now such as to contra-indicate operation, and she gradually sank and died on September $10 \mathrm{th}$.

Post mortem examination. The condition of the uterus will be described immediately. The only other part of the body in which growths were found was the thorax. The following note is taken from the post mortem book:-

"On opening the thorax a large mass of soft growth was seen occupying the region of the upper lobe of the right lung. It was loosely attached to lung and pleura and came away easily in the hand; it was the size of a large orange. On section it consisted of soft, brownish-red material, intersected by honeycomb-like structures, similar to the large mass in the uterus. Both lungs contained several smaller masses, dark in colour, some of which were easily cuucleated; on section they also reproduced closely the appearances of the uterine tumour."

The beart, liver and kidneys all showed evidence of fatty degeneration. No other lesions were found.

Description of the Specimen. The specimen consists of the left half of a uterus. The organ is enlarged, the part preserved measuring $8 \frac{1}{2}$ inches in length, $4 \frac{1}{2}$ inches antero-posteriorly and $2 \frac{3}{4}$ inches from side to side. The external surface, smooth and covered by peritoneum, presents a number of small bosses due to the presence of sub-peritoneal fibro-myomata.

At the lower part of the specimen is the cervix uteri, its canal is dilated, its lips are patulous, and the supra-vaginal portion is stretched and elongated to a length of $2 \frac{1}{4}$ inches; only faint traces of the arbor vitce are visible, but portions of the mucosa from the upper part of this canal have been submitted to microscopical examination and exhibit the characters of cervical not corporeal endometrium. At a 


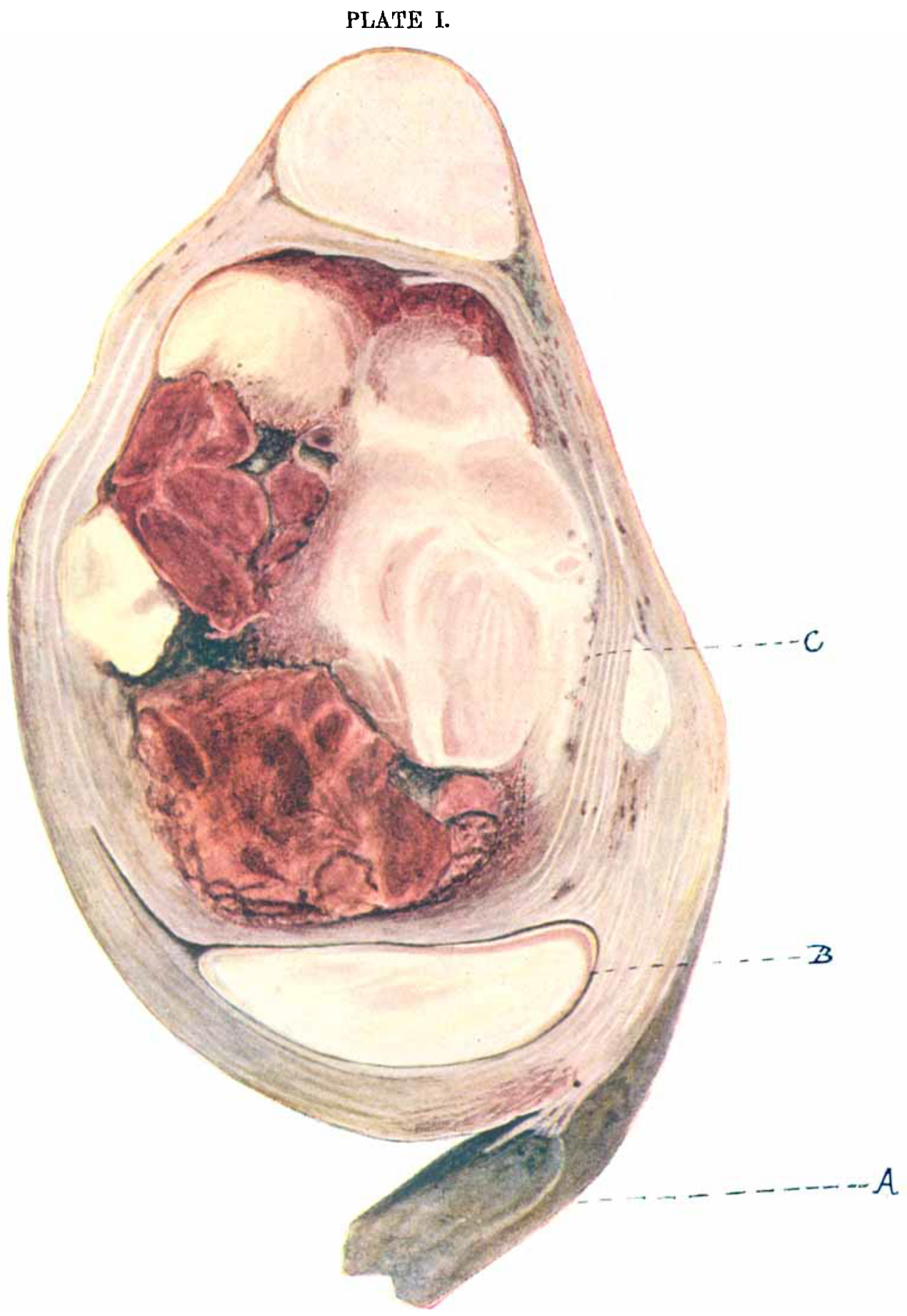

Sagittal, Shction through Uterus and Growth.

A.-Cervix utori.

B.-Uterine cavity containing fibroid polypus.

C.-Large interstitial growth in anterior wall of uterus, in part a fibronyoma, in part a sarcoma. 
spot on the external surface corresponding to the fundus uteri is the point of attachment of the Fallopian tube and of the round and ovarian ligaments. The round ligament is hypertrophied and elongated. The fimbriated end of the tube is closed and is closely adherent to the cystic ovary; a small opening has been made into the distal end of the tube and a glass rod passed along its lumen towards the uterus.

On section the greater part of the specimen is composed of a group of tumours growing in the anterior wall of the uterus. The uterine cavity lies at the lower part of the specimen, it is dilated and occupied by a fibroid polypus attached by a slender pedicle of mucous membrane to the anterior wall. The axis of the uterine cavity is almost at right angles to the cervical canal, and is directed backwards; the cavity is enlarged, measuring four and a half inches in length.

The walls of the uterus are hypertrophied; the posterior measures $\frac{5}{8}$ ths of an inch in thickness; the anterior contains interstitial tumours. One of these tumours occupies the highest part of the wall; it is the size of a golf ball, is encapsuled, and is composed of dense white tissue. Microscopical sections show that it is a fibro-myoma. A second growth, smaller, but possessing similar characters, lies lower down in the wall a short distance above the uterine cavity. The third tumour, much the largest of the three, presents very remarkable characters. It measures $5 \frac{1}{2}$ inches in length, $4 \frac{1}{2}$ inches antero-posteriorly; it is interstitial, surrounded everywhere by the fibro-muscular tissue which constitute the anterior wall of the uterus, and is composed of two varieties of growth:--

(a) Dense, white, fibrous-looking tissue, the continuity of which has become broken by the presence of

(b) Masses of friable material of a deep red or brown colour.

This latter material is situated chiefly in the posterior part of the tumour, and in its general appearance closely resembles blood-clot. Yicroseopic sections show that the dense white part of the tumour is a fibro-myoma, whilst the friable red portion is a sarcoma.

The fibroid polypus which occupies the uterine cavity can be easily displaced, it is then found that the cavity is everywhere lined by a smooth mucosa entirely free from new growth.

It is important to note once again that the whole mass (fibromyoma and sarcoma) is encapsuled, and whilst the sarcomatous elements have extensively invaded the fibro-myoma, as far as can be ascertained without mutilating the specimen, the uterine wall is but little affected. 
Description of Microscopical Appearances.

(1) Section from the firm white part of the tumour. The section presents the appearances commonly seen in a degenerate fibromyoma; it is composed of interlacing bands of unstriped muscle and of fibrous tissue. In some parts the nuclei still possess their usual staining reactions, in others they stain only feebly or not at all. The cell-fibres have lost their individual outlines and are represented by masses of almost homogeneous substance staining faintly with eosin. In some places are vacuolated areas and small cystic spaces formed by myxomatous degeneration of tissues, in other places are areas of inflammation with small-celled infiltration of the tissues. Only a few thin-walled vessels are seen scattered through the section. At one spot the fibro-myomatous tissues are invaded by an elongated strand of sarcoma cells. The characters of these cells are precisely similar to those to be described in the next section.

(2) Section from the red, friable part of the tumour. The friable portions of the tumour are composed of a mass of cells of whose malignancy there can be no question. They are of very different sizes and shapes, most of them are round, some are elongated and spindle-shaped, some are very large and contain as many as six or eight nuclei; these may be designated "giant cells." In nearly all the cells, the nuclei are large, active, and decply-staining, sometimes they occupy almost the whole of the cell. The cells are separated from one another by a homogeneous inter-cellular stroma which stains clearly with eosin. At the periphery of the malignant portion are elongated cells with long, rod-shaped nuclei, these cells lie near to the muscle cells of the fibro-myoma, and both can be seen in the same field of the microscope. They are obviously malignant, but in their shape and form resemble muscle cells; ${ }^{*}$ in most parts of the section, however, no such resemblance can be seen. No well formed blood-vessels are seen, but there are many vascular channels traversing the section.

(3) Sections from the secondary growths in the lungs. The cells in these masses are all of the round and spindle-shaped varieties. There are no cells visible which resemble muscle-cells in either their form or arrangement.

The study of the relation between sarcomata and fibro-myomata of the uterus is one which is beset with difficulties, and in which we must proceed with the greatest caution.

* It is important to pay attention to this point, for, as we shall see later, many pathologists hold that sarcoma of the uterus commonly arises from muscle-cells. 
PLATE II.

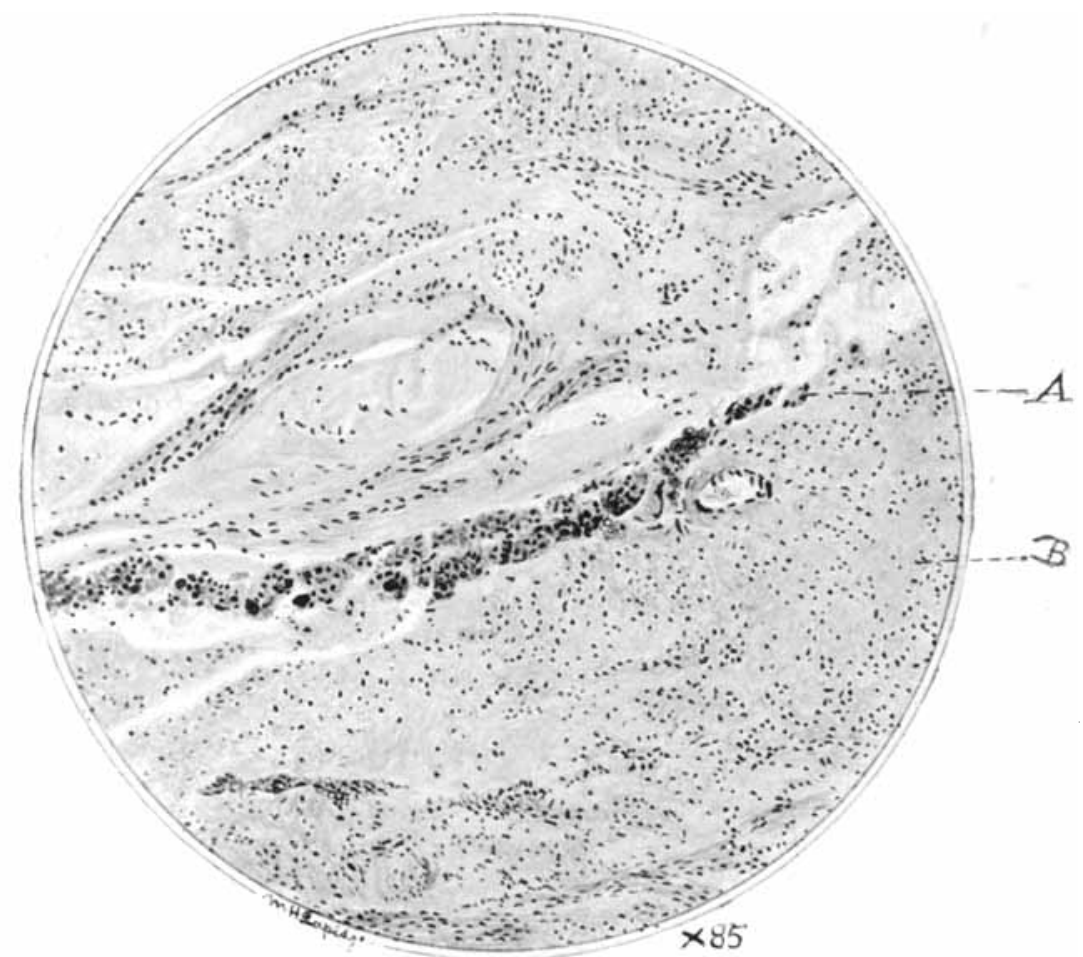

Section showing tissues of the fibro-myoma invaded by a strand of sarcoma cells. A, Sarcoma cells. B, Fibro-myoma. 
PLATE III.

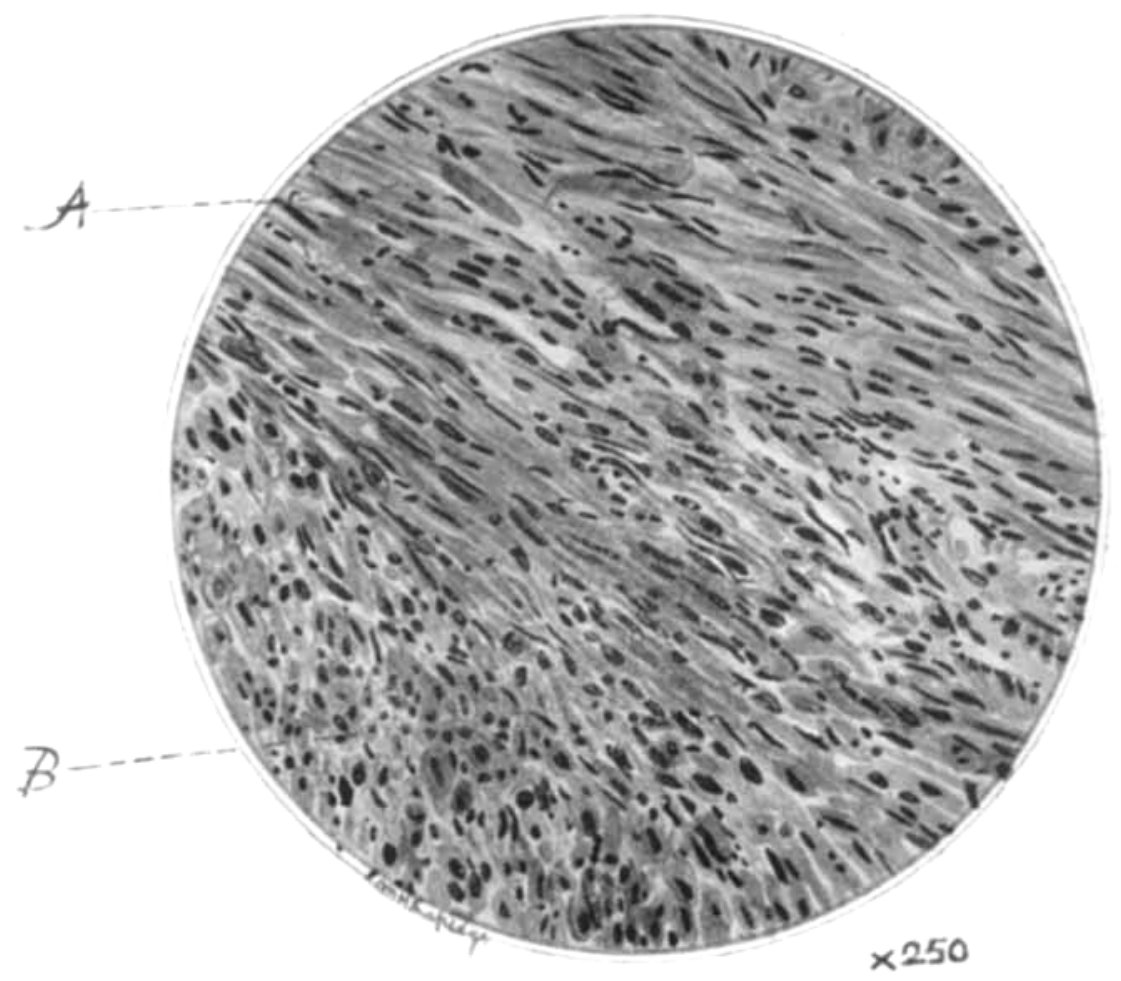

Section showing muscle bundles and sarcoma cells in the same field of the microscope. A, Muscle bundles. B, Sarcoma cells. 


\section{Griffth and Williamson: Sarcomatous Fibro-myoma 89}

The subject is rendered the more complicated by the use of the term "malignant degeneration" as applied to fibro-myomata. When an innocent tumour develops malignant characters we ought not to speak of the process as a degeneration; degeneration means a loss of vitality leading often through slow or more rapid gradations to ultimate death. Malignancy, on the contrary, implies an activity and vitality so great that along the track of the malignant invading cells the normal tissues are disintegrated and destroyed.

Further, different writers use this term, inappropriate as it is, to indicate very different conditions :-

(1) There may be present in the same uterus two entirely separate and distinct tumours, the one a sarcoma the other a fibro-myoma.

(2) A sareoma may originate in some more or less distant part of the uterine wall and subsequently invade a fibro-myoma.

(3) A sarcoma may arise de novo in a pre-existing fibro-myoma: a new growth within a new growth.

(4) Possibly the cells of which the existing fibro-myoma is constituted may assume malignant characters.

All four of these conditions have been described under the name of "malignant degeneration."

Many authors have positively stated or tacitly assumed that all sarcomata of the uterine parenchyma have their origin in fibromyomata. This doctrine, at one time widely accepted, has left its mark upon later writings, and even in recent papers there is oftcn no clear distinction made between primary sarcomata, and fibro-myomata undergoing a malignant change.

Is the association of sarcoma and fibro-myoma a common one? For the answer to this question we must turn to published statistics.

Dr. Cullingworth, in his admirably reported series of 100 fibromyomata, met with only one sarcoma.

Haultain, in a serics of 400 cases, met with one sarcoma. Simpson, in 300 cases, met with none; MacDonald, in 280 cases, with three. On the other hand, Mrs. Scharlieb, in 100 consecutive cases, met with 6 sarcomata, whilst Baker and Graves, out of a series of 33 cases, report 3 sarcomata. Here, then, in this town of London, the association appears to be six times as common in the practice of Mrs. Scharlieb, as it is in that of Dr. Cullingworth. What is the explanation? The diagnosis of sarcoma depends entirely upon the interpretation of certain histological appearances, one pathologist will accept these appearances as evidence of malignancy, another will 
refuse to do so. This fact is brought home to us very forcibly, if we review the cases of reputed sarcoma of the uterus brought before the Obstetrical Society of London during the last few years.

In 1902 Dr. Munro Kerr showed a small fibroid which, to quote his own words, exhibited "Groups of cells throughout the tumour which are not cross sections of muscle fibres. These are considered evidence of a sarcomatous change having occurred in this tumour."

The Pathology Committee reported:- "We can find no evidence of sarcomatous change in the tumour."

In $1901 \mathrm{Dr}$. Galabin showed a microscopic section of a tumour removed from an unmarried lady of 28. After removal the tumour was regarded as a fibro-myoma; a small piece was preserved for examination, the rest was thrown away. The Clinical Research Association reported it to be a sarcoma. Dr. Spencer differed from this view and regarded the section as part of a fibro-myoma. Unfortunately the specimen was not referred to a Committee.

In 1899 Dr. Amand Routh exhibited before the Society what he described as a myxo-sarcoma of the uterus. The Pathology Committee reported :- -

"We are of opinion that this specimen is a fibro-myoma, showing extensive round-celled infiltration (inflammatory) with general thrombosis of vessels and mucoid degeneration in parts."

These instances might be multiplied, but we have said enough to show how extremely difficult it is to arrive at any trustworthy conclusion regarding the frequency of the association. Mr. Doran has often pleaded the importance of after-histories-never with greater justice than in the case of these reputed sarcomata of the uterus.

The changes which may occur in a fibro-myoma as the result of œdema, of degeneration, and of inflammation, lead to marked alterations in both the muscle fibres and the connective-tissue cells, alterations which are often interpreted as evidences of malignancy.

In the case of the specimen before us there can be no question that we are dealing with a true sarcoma. Its constituent cells have an the characters which we associate with malignancy; the growth has unquestionably invaded surrounding tissues; metastatic deposits of a similar structure are found in distant organs, and, finally, the disease has killed the patient.

To determine what is the relation of the sarcoma to the fibromyoma is a matter of grave difficulty, and the discussion of this question will necessitate a brief review of certain other cases.

We have already drawn attention to the fact that sarcoma and 
fibro-myoma may co-exist in the same uterus under four different conditions. Let us examine each of these a little more closely.

(1) The two growths may exist as separate and distinct tumours. For the proof of this statement we need only refer to a specimen exhibited before the Obstetrical Society on May 7 th, 1902, by Dr. Tate, and figured on Plate IVa of the Society's Transactions for that year. The specimen was referred to a Pathology Committee, of which one of us was a member. The report was as follows :

"We are of opinion that this specimen is a primary sareoma of the body of the uterus, and that it is associated with a fibro-myoma. There is no evidence that the sarcoma represents a malignant degeneration of a fibro-myoma. There is a distinct line of demarcation between the two tumours. The microscopical appearances show that the malignant tumour is a mixed, round and spindle-celled, sarcoma, whilst the innocent tumour is undoubtedly a fibro-myoma. There is no evidence of invasion of the fibro-myoma by the sarcomatous growth."

It is quite clear that the specimen before us cannot be referred to this class.

(2) A sareoma may originate in a more or less distant part of the uterus, and subsequently invade a pre-existing fibro-myoma. For the proof of this statement we select a case published by Whitridge Williams in the American Journal of Obstetrics, vol. xxix., p. 753. Similar cases have been published by Raymond (Progres Médical, vol. ix., p. 741) and Gottschalk (Central. für Gynäkol., vol. xi., p. 242). Williams's description is as follows:--

"At the fundus, the uterine wall was one centimeter thick, its lower portion being of various thickness, for a nodular tumour four centimeters in diameter occupied its anterior wall and extended down to the os internum. The surface of the tumour projected into the uferine cavity and presented a jagged, irregular, ulcerated appearance. The rest of the uterine cavity showed no trace of its normal mucous membrane, but presented an irregular villous necrotic surface which was composed of a soft tissuc rich in vesscls, and which apparently extended down into the muscularis."

Microscopic sections from this irregular villous growth showed it to be a spindle and round-celled sarcoma containing some giant cells.

"Sections from various parts of the specimen clearly show that the sarcomatous new growth is limited to the inner surface of the uterus and extends only a short distance into its walls. It is probable 
that it originated in the mucosa, but as no trace of the endometrium remains, positive proof for this mode of origin cannot be adduced. Sections through the upper part of the large myomatous nodule and the adjacent portions of the uterine wall and cavity, show clearly that the growth did not originate in the myoma, but only involved the portions of it which were adjacent to the uterine cavity, just as it did the other portions of the uterine wall."

It is a fact worthy of note that in the three similar cases of which we have found records the sarcoma appears to have had its origin in the endometrium.

Can we place the specimen before us in this group of cases? We believe not-certainly the sarcoma did not arise in the endometrium, for this structure is everywhere intact, and the examination of microscopic soctions reveals no pathological change beyond some flattening and atrophy due probably to pressure. Nor can we find any evidence to justify the view that it arose in the fibro-muscular wall of the uterus; we have examined sections of the uterine wall in the immediate vicinity of the tumour, and find them free from ovidence of malignant disease.

We are compelled then to place this tumour in one of the two last groups we have enumerated; and in our opinion, the present state of our knowledge of the histo-genesis of malignant tumours in general does not pormit us to distinguish definitely between themn.

We find no difficulty whatever in accepting the doctrine that a tumour, originally innocent in both its clinical characters and microscopic appearances, may become malignant: but whether the cells of the existing tumour assume malignant characters, or whether the malignant growth arises de novo in the innocent tumour and by its invasion destroys and replaces the tissues of its host, we cannot say.

A fibro-myoma is composed of two varicties of tissue, muscle cells and connective-tissue cells, and in most cases it is from one or other of these forms of tissue that the cells of the sarcomata are derived. We say "in most cases," for in a few rare instances the growths have arisen from lining cells of blood-vessels or of lymph channels. These cases of uterine endotheliomata are however, admittedly, few and far between.

Has the sarcoma arisen from muscle or from connective-tissue cells?

Many authors have described a malignant change occurring in muscle cells. To the form of tumour thus constituted the term. 
"leiomyoma malignum" has been applied. Clinically these tumours are distinguished by their rapid growth, by their tendency to recur after removal, and by the development of metastases of similar structure in the lungs and other viscera. On microscopic examination the cells are seen to be elongated and of many varieties. Tlesko-Straganova, von Kehlden and Whitridge Williams, state that they have traced every stage of the transition from an apparently normal unstriped muscle-fibre on the one hand to a surcoma spindle-cell on the other. Round cells and giant cells, often containing many nuclei, are commonly met with, and in general it is only at the periphery of the growth that the rescmblance to muscle-fibres can be traced.

German and American pathologists regard the muscle cell as the commoner site of origin of the malignant growth; on the other hand the view of the members of the Obstetrical Society of London has hitherto been that sarcoma arises only from the connective-tissue cells. This is the view expressed by Dr. Horrocks, and recorded in the Society's Transactions for 1898, p. 178, and again in the Transactions for 1901, p. 226, and at neither of the meetings were his opinions controverted.

We have pointed out in our description of the microscopic appearances of this specimen, the resemblance in shape and form, at the growing edge of the tumour, between the sarcoma cells and the adjacent muscle-fibres. The cells are elongated and pointed at each end; the nuclei, enormously enlarged and in process of active division, are in some cells still rod-shaped, in others rounded, and further the cells are still grouped together in the form of bundles and strands. These appearances certainly suggest that the sarcoma may have arisen from muscle-fibres. Other cases have been described which render equally probable a connective-tissue origin, and the truth is that from either of these cells a malignant growth may arise. We see no need to introduce a new term such as "leiomyoma malignum," both the tissues are of mesoblastic origin and a malignant neoplasm of either is correctly described as a sarcoma.

We regard the specimen as an instance of sarcomatous change occurring in a fibro-myoma. Whether the sarcoma has arisen de novo in a pre-existing fibro-myoma, or whether the cells of the fibro-myoma have assumed malignant characters, we do not pretend to say. Further, from the microscopical appearances seen at the growing edge of the tumour, we think it quite possible that the sarcoma may have, at least in part, originated in muscle cells. 


\section{ReFERENCES.}

1. Cullingworth, "An analysis of 100 cases of fibro-myoma," Jour. Obs. and Gyn. Brit. Emp., 1902.

2. Baker and Graves, Amer. Jour. Obstet., Sept., 1903.

3. McDonald, "Complications and Degenerations of Uterine Myoma," Jour. Am. Med. Assoc., 1904.

4. Haultain, "Malignant complications of Fibro-myromata," Jour. Ots. and Gyn. Brit. Emp., 1904.

5. Scharlieb, "Analysis of 100 cases of Fibro-myomata," Jour. Obs. and Gyn. Brit. Emp., 1902, Vol. II.

6. Ulesko-Stroganova, "Ueber das maligne Myom," Monats. für Geb. und Gyn., XVIII., H. 3 and 4.

7. Von Kehlden, Ziegler's Beiträge, XIV., 1893.

8. Whitridge Williams, Amer. Jour. Obs., Vol. 29, p. 721. 